\title{
MicroRNA basis of physiological hypertrophy
}

\author{
Siyi Fu ${ }^{1,2+}$, Ran Zhuo ${ }^{1,2+}$, Mengchao Yao ${ }^{1,2+}$, Jiawen Zhang ${ }^{1,3}$, Hanling Zhou ${ }^{1}$ and Junjie Xiao ${ }^{1,2,3 *}$ \\ ${ }^{1}$ Regeneration Lab, School of Life Science, Shanghai University, Shanghai, China \\ 2 Innovative Drug Research Center of Shanghai University, Shanghai, China \\ ${ }^{3}$ Experimental Center of Life Sciences, School of Life Science, Shanghai University, Shanghai, China \\ *Correspondence: junjiexiao@live.cn \\ Edited by: \\ Yi-Qing Yang, Shanghai Chest Hospital and Medical College of Shanghai Jiaotong University, China \\ ${ }^{t}$ These authors have contributed equally to this work.
}

Keywords: microRNAs, pathological hypertrophy, physiological hypertrophy, proliferation, heart failure

Cardiac hypertrophy is a distinguished feature of several physiological and pathological remodeling (Frey et al., 2004). Pathological hypertrophy is commonly seen in patients with heart injury or stress, such as myocardial infarction, hypertension, and valve disease (Frey et al., 2004). Physiological hypertrophy is typically induced by exercise or pregnancy. Interestingly, physiological hypertrophy is generally accepted as an adaptive beneficial response while pathological hypertrophy can ultimately decompensate to heart failure, a common, costly, disabling, and deadly disease. Thus, dissecting the mechanisms for physiological hypertrophy will help identify novel effective therapies for a large spectrum of cardiovascular diseases (Da Costa Martins and De Windt, 2012).

Distinct underlying signaling pathways for physiological and pathological hypertrophy have been identified (Maillet et al., 2013). The classic pathway for physiological hypertrophy is IGF-1/PI3K (p110a)/Akt1 while the key one for pathological hypertrophy is AngII (ET1)/Gaq/Calcineurin/NFAT (Maillet et al., 2013). MicroRNAs (miRNAs, miRs) are a novel class of non-coding RNAs with 20-24 length of base, which posttranscriptional regulates gene expression via base-pairing with complementary sequences within mRNA (Xiao et al., 2012). It is estimated that over 1000 miRNAs were encoded by the human genome. Individual miRNAs can regulate several target genes while one gene can also be regulated by several miRNAs. Being a center player of gene regulation, miRNAs participate in many essential biological processes, including proliferation, differentiation, apoptosis, necrosis, autophagy, and stress responses (Xiao et al., 2012; Kumarswamy and Thum, 2013). Due to these multiple roles, it is naturally that miRNAs are critical in the development of various heart diseases, such as hypertrophy, heart failure, acute myocardial infarction, and arrhythmia (Xiao et al., 2011; Kumarswamy and Thum, 2013). In addition, circulating miRNAs have also been indicated to be promising biomarkers for cardiovascular diseases (Xu et al., 2012). Among them, pathological hypertrophy is the most widely studied one. Accumulating evidence has indicated that a lot of miRNAs such as miR-1, miR-133, miR-26, miR-9, miR-98, miR-29, miR-199a, miR-199b, miR-208, miR-23a, miR-499, miR-21, and mir-19b contribute to pathological hypertrophy (Da Costa Martins and De Windt, 2012). Some distinguished reviews have summarized it in detail (Da Costa Martins and De Windt, 2012; Ellison et al., 2012).

Unlike pathological hypertrophy, only a little studies described how miRNAs response to physiological hypertrophy (Soci et al., 2011; Diniz et al., 2013). It has been reported that miR1, miR-133, mir-29c, miR-27a, mir-27b, and miR-143 response to physiological hypertrophy (Soci et al., 2011; Da Costa Martins and De Windt, 2012; Ellison et al., 2012). However, these miRNAs are either compensatory or lack of direct evidence for regulating cell size. Thus, these results might only set the beginning of filling the gap in miRNAs and physiological hypertrophy. Although these studies have suggested some potential roles of miRNAs in physiological hypertrophy, more functional studies are highly needed to establish
miRNAs as contributors for physiological hypertrophy.

Traditionally, the adult mammalian heart is recognized as a post-mitotic organ with no regenerative capacity for cardiomyogenesis (Rosenzweig, 2012). Recently, resident endogenous cardiac stem-progenitor cells (eCSCs) in the adult heart challenged this dogma (Rosenzweig, 2012). Moreover, adult cardiomyocytes have also been reported to proliferate in response to specific stimuli (Rosenzweig, 2012). Two studies regarding physiological hypertrophy are of great importance (Boström et al., 2010; Waring et al., 2012). A transcriptional factor named CEBPB has been found to be down-regulated with exercise and reduction of CEBPB induces cardiomyocyte hypertrophy and proliferation (Boström et al., 2010). Further studies show that CEBPB promotes cardiomyocyte proliferation via increasing CITED4 (Boström et al., 2010). This study indicates that besides the generally accepted idea that physiological hypertrophy is solely due to the hypertrophy of existing cardiomyocytes, physiological hypertrophy also has the phenotype of new cardiomyocytes formation (Boström et al., 2010). A more recent study shows that $\mathrm{c}-\mathrm{Kit}$ positive eCSCs increases their number and activated state in exercise-induced physiological hypertrophy, indicating that $\mathrm{c}$-Kit positive eCSCs might be a source of new cardiomyocyte formation (Waring et al., 2012). Therefore, myocyterestricted lineage tracing studies are highly needed to definitively unravel this question. Anyway, both studies indicate that it is necessary to check miRNAs roles in promoting new cardiomyocyte formation either in cardiomyocytes or in eCSCs in physiological hypertrophy. 
With the strategies for checking myocyte hypertrophy and new cardiomyocyte formation, the miRNA basis of physiological hypertrophy will be revealed, which will help develop a miRNA-based therapy for heart failure.

\section{ACKNOWLEDGMENTS}

This work was supported by the grants from National Natural Science Foundation of China (81200669 to J. Xiao), Innovation Program of Shanghai Municipal Education Commission (13YZ014 to J. Xiao), Foundation for University Young Teachers by Shanghai Municipal Education Commission (year 2012, to J. Xiao), Innovation Foundation of Shanghai University (sdcx2012038, to J. Xiao), National Undergraduate Training Programs for Innovation and Entrepreneurship (year 2012, to J. Zhang), and Shanghai Undergraduate Training Programs for Innovation and Entrepreneurship (year 2012, to H. Zhou).

\section{REFERENCES}

Boström, P., Mann, N., Wu, J., Quintero, P. A., Plovie, E. R., Panáková, D., et al. (2010). C/EBPbeta controls exercise-induced cardiac growth andprotects against pathological cardiac remodeling. Cell 143, 1072-1083. doi: 10.1016/j.cell.2010.11.036

Da Costa Martins, P. A., and De Windt, L. J. (2012). MicroRNAs in control of cardiac hypertrophy. Cardiovasc. Res. 93, 563-572. doi: $10.1093 / \mathrm{cvr} / \mathrm{cvs} 013$

Diniz, G. P., Takano, A. P., and Barreto-Chaves, M. L., (2013). MiRNA-208a and miRNA-208b are triggered in thyroidhormone-induced cardiac hypertrophy - role of type 1 Angiotensin II receptor (AT1R) on miRNA-208a/alpha-MHC modulation. Mol. Cell. Endocrinol. 374, 117-124. doi: 10.1016/j.mce.2013.04.010

Ellison, G. M., Waring, C. D., Vicinanza, C., and Torella, D. (2012). Physiological cardiac remodelling in response to endurance exercise training: cellular and molecular mechanisms. Heart 98 , 5-10. doi: 10.1136/heartjnl-2011-300639

Frey, N., Katus, H. A., Olson, E. N., and Hill, J. A., (2004). Hypertrophy of the heart: a new therapeutic target? Circulation 109, 1580-1589. doi: 10.1161/01. CIR. 0000120390.68287.BB

Kumarswamy, R., and Thum, T. (2013). Noncoding RNAs in cardiac remodeling and heart failure. Circ. Res. 113, 676-689. doi: 10.1161/CIRCRESAHA.113.300226

Maillet, M., van Berlo, J. H., and Molkentin, J. D. (2013). Molecular basis of physiological heart growth: fundamental concepts and new players. Nat. Rev. Mol. Cell Biol. 14, 38-48. doi: $10.1038 / \mathrm{nrm} 3495$

Rosenzweig, A. (2012). Medicine. Cardiac regeneration. Science 338, 1549-1550. doi: 10.1126/science. 1228951

Soci, U. P., Fernandes, T., Hashimoto, N. Y., Mota, G. F., Amadeu, M. A., Rosa, K. T., et al. (2011). MicroRNAs 29 are involved in the improvement of ventricular compliance promoted by aerobic exercise training in rats. Physiol. Genomics 43, 665-673. doi: 10.1152/physiolgenomics.00145

Waring, C. D., Vicinanza, C., Papalamprou, A., Smith, A. J., Purushothaman, S., Goldspink, D. F., et al. (2012). The adult heart responds to increased workload with physiologic hypertrophy, cardiac stem cell activation, and new myocyte formation. Eur. Heart J. doi: 10.1093/eurheartj/ehs338. [Epub ahead of print].

Xiao, J., Liang, D., Zhang, H., Liu, Y., Zhang, D., Liu, Y., et al. (2012). MicroRNA-204 is required for differentiation of human-derived cardiomyocyte progenitor cells. J. Mol. Cell. Cardiol. 53, 751-759. doi: 10.1016/j.yjmcc.2012.08.024

Xiao, J., Liang, D., Zhang, Y., Liu, Y., Zhang, H., Liu, Y., et al. (2011). MicroRNA expression signature in atrial fibrillation with mitral stenosis. Physiol. Genomics 43, 655-664. doi: 10.1152/physiolgenomics.00139.2010

Xu, J., Zhao, J., Evan, G., Xiao, C., Cheng, Y., and Xiao, J. (2012). Circulating microRNAs: novel biomarkers for cardiovascular diseases. J. Mol. Med. (Berl.) 90, 865-875. doi: 10.1007/s00109-0110840-5

Received: 28 October 2013; accepted: 07 November 2013; published online: 26 November 2013.

Citation: Fu S, Zhuo R, Yao M, Zhang J, Zhou H and Xiao J (2013) MicroRNA basis of physiological hypertrophy. Front. Genet. 4:253. doi: 10.3389/fgene.2013.00253 This article was submitted to Epigenomics and Epigenetics, a section of the journal Frontiers in Genetics.

Copyright (C) 2013 Fu, Zhuo, Yao, Zhang, Zhou and Xiao. This is an open-access article distributed under the terms of the Creative Commons Attribution License (CC BY). The use, distribution or reproduction in other forums is permitted, provided the original author(s) or licensor are credited and that the original publication in this journal is cited, in accordance with accepted academic practice. No use, distribution or reproduction is permitted which does not comply with these terms. 